\title{
Whole genome sequencing based noninvasive prenatal test
}

\author{
Eun-Hae Cho* \\ Green Cross Genome, Yongin, Korea
}

\begin{abstract}
Whole genome sequencing (WGS)-based noninvasive prenatal test (NIPT) is the first method applied in the clinical setting out of various NIPT techniques. Several companies, such as Sequenom, BGI, and Illumina offer WGS-based NIPT, each with different technical and bioinformatic approaches. Sequenom, BGl, and Illumina utilize $z-, t-$, and $L$-scores, as well as normalized chromosome values, respectively, for trisomy detection. Their outstanding performance has been demonstrated in clinical studies of more than 100,000 pregnancies. The sensitivity and specificity for detection of trisomies 13, 18, and 21 were above $98 \%$, as reported by all three companies. Unlike other techniques, WGS-based NIPT can detect other trisomies as well as clinically significant segmental duplications/deletions within a chromosome, which could expand the scope of NIPT. Incorrect results could be due to low fetal fraction, fetoplacental mosaicism, confined placental mosaicism or maternal copy number variation (CNV). Among those, maternal CNV is a significant contributor of false positive results and therefore genome wide scanning plays an important role in preventing the occurrence of false positives. In this article, the bioinformatic techniques and clinical performance of three major companies are comprehensively reviewed.
\end{abstract}

Key words: Noninvasive prenatal test, Whole genome sequencing, Down syndrome, Trisomy 18, Patau syndrome.

\section{Introduction}

Whole genome sequencing (WGS)-based noninvasive prenatal test (NIPT) is the first clinically introduced method among various NIPT techniques. WGS-based NIPT is now offered by several companies such as Sequenom (San Diego, CA, USA), BGI (Shenzhen, China), Illumina (Redwood City, CA, USA), and Berry Genomics (Beijing, China). The MaterniT2 $1{ }^{\circledR}$ Plus test developed by Sequenom provides information about trisomies $13,16,18,21$, and 22 as well as certain sex chromosome aneuploidies and some microdeletions [1]. The Visibilit ${ }^{\mathrm{TM}}$ test, a lower cost NIPT provided by Sequenom, focuses detection on only trisomies 18 and 21 for average risk pregnancies [2]. The
NIFTY ${ }^{\mathrm{TM}}$ test by BGI detects trisomies 13, 18, and 21 as well as some sex chromosome aneuploidies and selected microdeletions [3]. Verinata Health, a subsidiary of Illumina offers the Verifi ${ }^{\circledR}$ test for detection of trisomies 13,18,21, and the presence of monosomy $X$. The expanded version of this test also detects other aneuploidies and microdeletions [4]. Compared to other NIPT techniques such as the targeted sequencing method (either chromosome selective or single nucleotide polymorphism based) and those that use differences in methylation patterns between maternal and fetal DNA, WGS-based NIPT has an advantage in scanning genome wide abnormalities. Therefore, WGS-based NIPT companies are putting significant effort into improving the accuracy of tests that detect clinically relevant

Received: 23 July 2015, Revised: 13 November 2015, Accepted: 13 November 2015, Published: 31 December 2015

${ }^{*}$ Corresponding author: Eun-Hae Cho, M.D., Ph.D.

Green Cross Genome, 107 Ihyeon-ro 30 beon-gil, Giheung-gu, Yongin 16924, Korea.

Tel: +82-31-260-9216, Fax: +82-31-260-0620, E-mail: ehcho@greencross.com

Conflict of interest: Green Cross Genome is developing WGS-based NIPT.

(ac) This is an open-access article distributed under the terms of the Creative Commons Attribution Non-Commercial License (http://creativecommons.org/licenses/by-nc/4.0/) which permits unrestricted non-commercial use, distribution, and reproduction in any medium, provided the original work is properly cited.

(c) Copyright 2015 by the Korean Society of Medical Genetics 
deletions and duplications.

The clinical performance of these tests has been reported by many validation studies and all demonstrate high levels of sensitivity and specificity [5-9]. BGI has evaluated over 400,000 NIFTY samples derived from all over the world and published these findings from the largest clinical study which includes 146,958 pregnancies [5]. Sequenom also reported that they performed over 250,000 commercial tests to date [1] as well as tested the clinical performance of over 100,000 samples [7]. Despite the excellent clinical performance reported by NIPT companies, incorrect results continue to be obtained. Therefore, these companies aim to improve the technical and bioinformatic methods used in order to minimize false negative and positive results despite the biological limitations on testing cell free DNA, not fetal DNA itself. In this article, the current clinical services and technical methods used by three major WGS-based NIPT companies (Sequenom, BGI, and Illumina) are presented. Additionally, a comparative analysis of each company's technical and clinical performance is outlined (Table 1).

\section{Clinical Services and Technical Methods Used in WGS-based NIPT}

\section{Sequenom: MaterniT21 ${ }^{\circledR}$ Plus}

Sequenom currently requires $20 \mathrm{~mL}$ of whole blood, and an average 5-day turnaround time from receipt of sample in the laboratory [1]. The test report includes identification of trisomies 13,18 , and 21 as well as fetal gender. Fetal sex chromosomal abnormalities, rare fetal trisomies like 16 and 22, and selected microdeletions including 22q (DiGoerge syndrome), $15 q$ (Prader-Willi/Angelman syndrome), 11q (Jacobsen syndrome), $8 q$ (Langer-Giedion syndrome), $5 p$ (cri du chat syndrome), $4 p$ (Wolf-Hirschhorn syndrome), and 1 p36 deletion syndromes are reported when detected. The Materni21Plus ${ }^{\mathrm{TM}}$ test can be used as early as 10 weeks' gestation and can be applied to multiple pregnancies. Circulating cell-free DNA was extracted using the OlAamp circulating nucleic acid kit (Qiagen, Valencia, CA, USA), and the fetal fraction was quantified by the fetal quantifier assay which uses methylation-based DNA discrimination with matrix assisted laser desorption ionization time of flight mass spectrometry (MALDI-TOF MS) [10]. Indexed sequencing

Table 1. Comparison of the technical and clinical performances between commercial noninvasive prenatal test

\begin{tabular}{|c|c|c|c|}
\hline & Sequenom & $\mathrm{BGl}$ & Verinata Health \\
\hline Test name $[1,3,4]$ & MaterniT21 & NIFTY & Verifi \\
\hline Test contents $[1,3,4]$ & $\begin{array}{l}\text { T21, T18, T13, T16, T22, SCA, } \\
22 q, 5 p, 15 q, 1 p 36,4 p, 8 q, 11 q\end{array}$ & $\begin{array}{l}\text { T21, T18, T13, SCA, 5p, 1p36, } \\
\text { 2q33.1 }\end{array}$ & $\begin{array}{l}\text { T21, T18, T13, SCA, optional tests } \\
\text { for microdeletion and other } \\
\text { chromosomal aneuploidies }\end{array}$ \\
\hline Test for multiple gestation $[1,3,4]$ & Yes & Yes & Yes \\
\hline Earliest gestational age $[1,3,4]$ (week) & 10 & 10 & 10 \\
\hline Market entry $[1,3,4]$ & October 2011 & August 2011 & March 2012 \\
\hline $\begin{array}{l}\text { Bioinformatic assessment of trisomies } \\
{[6,12,13,18,19]}\end{array}$ & $\begin{array}{l}\text { T21 z-score }>3 \\
\text { T18 and } 13 \\
z \text {-score }>3.95\end{array}$ & $\begin{array}{l}t \text {-score }>2.5 \\
L \text {-score }>1\end{array}$ & $\mathrm{NCV}>4$ \\
\hline $\begin{array}{l}\text { Pregnancies of the largest study } \\
{[5,7,9]}\end{array}$ & 100,000 & 112,669 & 34,306 \\
\hline Sensitivity $[5,7,9](\%)$ & $\begin{array}{l}\text { T21 }(99.6) \\
\text { T18 }(99.0) \\
\text { T13 }(98.9)\end{array}$ & $\begin{array}{l}\text { T21 (99.2) } \\
\text { T18 (98.2) } \\
\text { T13 (100.0) }\end{array}$ & $\begin{array}{l}\text { T21 (99.1) } \\
\text { T18 (98.3) } \\
\text { T13 (98.9) }\end{array}$ \\
\hline Specificity $[5,7,9](\%)$ & $\begin{array}{l}\text { T21 }(99.9) \\
\text { T18 }(99.9) \\
\text { T13 }(99.9)\end{array}$ & $\begin{array}{l}\text { T21 (99.9) } \\
\text { T18 (99.9) } \\
\text { T13 (99.9) }\end{array}$ & $\begin{array}{l}\text { T21 }(99.9) \\
\text { T18 }(99.9) \\
\text { T13 }(99.8)\end{array}$ \\
\hline $\begin{array}{l}\text { False negatives }[5,7,9] \\
\text { (number of cases) }\end{array}$ & $\begin{array}{l}\text { T21 (6) } \\
\text { T18 (5) } \\
\text { T13 (2) }\end{array}$ & $\begin{array}{l}\text { T21 (6) } \\
\text { T18 (3) } \\
\text { T13 (0) }\end{array}$ & $\begin{array}{l}\text { T21 (5) } \\
\text { T18 (3) } \\
\text { T13 (1) }\end{array}$ \\
\hline Test failure rate $[5,7,9](\%)$ & 1.3 & 0.098 & 0.1 \\
\hline
\end{tabular}

T21, trisomy 21; T18, trisomy 18; T13, trisomy 13; T16, trisomy 16; T22, trisomy 22; SCA, sex chromosome aneuploidy.

$z$-score, normalized value of percent sequence counts mapped to target chromosome adjusted by those results of euploid samples; $t$-score, normalized values of sequencing coverage adjusted by those results of euploid samples by GC correlation $t$-test; $L$-score, logarithmic likelihood ratio of $t$-scores for binary hypothesis ( $t$-score for first hypothesis/t-score for second hypothesis; first hypothesis: $\mathrm{HO}=$ =euploid, $\mathrm{H} 1=$ =trisomy; second hypothesis: $\mathrm{HO}=$ =trisomy, $\mathrm{H1}$ =euploid). NCV, normalized chromosomal value of sequenced reads adjusted by those results of internal chromosome controls (the sum of chr 2 to 6 for analyzing trisomy 13 and chr $Y$, chr 6 for analyzing chr X, chr 8 for analyzing trisomy 18, and chr 9 for analyzing trisomy 21). 
libraries are multiplexed and sequenced for 36 cycles on the HiSeq 2000 (Illumina, San Diego, CA, USA) [7]. Following sequencing, sequence reads that were uniquely mapped to specific chromosomes were counted and divided by all sequence reads excluding sequence reads from chromosome $X$ and $Y$ [11]. The percentage of specific chromosomes was then converted to $z$-score by subtracting the mean of the control group and dividing by the standard deviation of the same control group. The control group was a set of known euploid reference samples. $z$-score at or above 3 at chromosome 21 is considered to be indicative of Down syndrome and $z$-scores at or above 3.95 are considered indicative of trisomies 13 and $18[12,13]$. For detection of sex chromosomal aneuploidies, Sequenom developed an algorithm that first classifies female and male pregnancies, then the ratios of normalized chromosome $X$ and $Y$ read counts in the specific genomic regions versus the total autosomal read counts are determined. $z$-scores below -3.5 or above 3.5 at chromosome $X$ are indicative of Turner syndrome or XXX, respectively [14]. Samples with fetal fraction of $<4 \%$ or $>50 \%$ were rejected, and post-sequencing quality control criteria required a minimum of 9 million autosomal aligned reads [14]. Recently, SeqFF, a novel method for estimating fetal fraction in the plasma of pregnant women using sequence read counts was introduced [15].

\section{BGI: NIFTY'M}

$\mathrm{BGI}$ uses $5 \mathrm{~mL}$ of whole blood sample, which is comparably a small amount of blood, for analysis as early as 10 weeks' gestation [5]. Results are available within 10 working days and NIFTY'M detects trisomies 13, 18, and 21 as well as sex chromosomal aneuploidies and deletions (cri du chat syndrome, 1p36, 2q33.1) [2]. Thirty-six cycles of single end sequencing was performed using Hiseq 2000. BGI insists that the coefficient of variation for the standard $z$-score approach is larger than that of other approaches, especially for chromosomes 13 and 18. Therefore, they introduced a GC correlation and developed a binary hypothesis strategy to achieve a higher sensitivity and specificity [16]. A GC correlation t-test and logarithmic likelihood ratio ( $L$-score) between two $t$-tests, based on null/alternative hypotheses, was used to detect fetal trisomies 13, 18, and 21 [5]. If the $t$-score was $<2.5$ and the $L$-score $<1$, the sample was reported as negative, and if the $t$-score was $>2.5$, the sample was reported as positive with additional $L$-score information ( $L$-score $<1$ [warning zone] and $L$-score $>1$ [high risk zone]) [6]. For detection of sex chromosomal aneuploidies, BGI initially performed fetal gender identification using an experimental threshold and logistic regression. A t-test was then conducted if the fetus is female [16]. For a male fetus, a $t$-score was calculated to detect $X X Y$ and $X Y Y$, then fetal fractions were estimated by chromosome $X$ and $Y$, respectively. For an $X X Y$ sample, the fetal fraction estimated by chromosome $X$ was nearly zero and for $X Y Y$ sample, the $R$-value (ratio of the fetal fraction estimated by chromosome $Y$ to that estimated by chromosome $X$ ) was nearly two [16]. Fetal fraction was estimated, based on parent-specific homozygous single nucleotide polymorphism for discrepant results [17]. The minimum requirement for the fetal fraction was $>3.5 \%$ and post-sequencing quality control criteria required a minimal amount of unique sequencing reads of 2 million after alignment, which corresponded to about 5 million raw sequencing reads [5].

\section{Illumina: Verifi ${ }^{\circledR}$}

Illumina requires $7-10 \mathrm{~mL}$ of whole blood with an average turnaround time of 5.1 business days after receipt of the sample [18]. The basic Verifi ${ }^{\circledR}$ test screens for trisomies 13, 18, and 21 as well as the sex chromosome test option for monosomy $X$. Optional tests for microdeletions and other aneuploidies are also available. Plasma DNA is extracted using the OIAamp DNA Blood Mini Kit (Qiagen) and sequencing libraries are prepared using the Illumina TruSeq kit v2.5. Sequencing is performed on an Illumina HiSeq 2000 instrument and single-end reads of 36 base pairs are obtained [8]. The Verifi ${ }^{\circledR}$ test introduces the normalized chromosome value (NCV) algorithm to minimize the variations in and between sequencing runs. Unique reads are mapped and counted for calculation of the NCV in which denominators are cumulative counts on predetermined sets of optimal chromosomes that minimize the variation by calculating all possible permutations of denominators for all autosomes and sex chromosomes [19]. If the NCV of specific chromosomes is more than 4 , the sample is regarded as aneuploid for that chromosome. For detection of sex chromosome aneuploidies, normalized chromosome values for both $X$ and $Y$ is analyzed. If the sample is classified as female and euploid, the presence of fetal DNA is tested using the AmpfISTR Minifiler kit (Life technologies, Carlsbad, CA, USA) to find the minor peaks that represent fetal DNA. In the case of no minor peaks, a 15 single nucleotide polymorphisms panel is used [8].

\section{Clinical Performance of WGS-based NIPT}

\section{Sequenom: MaterniT21 ${ }^{\circledR}$ Plus}

Sequenom evaluated the clinical experience during 100,000 
tests and estimated clinical performance based on the clinical feedback of the confirmed discordant results [7]. The average maternal and gestational ages were 35.1 years and 15 weeks 3 days, respectively. The estimated sensitivity and specificity were 99.6\% and 99.9\% for trisomy $21,99 \%$ and $99.9 \%$ for trisomy 18 , and $98.9 \%$ and $99.9 \%$ for trisomy 13 . For 3,530 multiple gestations, the sensitivity and specificity were $>99.9 \%$ and 99.9\% for trisomy 21 , respectively, $95.20 \%$ and $>99.9 \%$ for trisomy 18 , respectively, and $>99.9 \%$ and $99.9 \%$ for trisomy 13 , respectively. There were 24 false positives ( 5 for trisomy 21, 5 for trisomy 18 and 14 for trisomy 13) and 13 false negatives ( 6 for trisomy 21,5 for trisomy 18 , and 2 for trisomy 13 ). According to the data shown above, the positive predictive value of trisomies 21,18 , and 13 could be estimated as $99.7 \%(1,510 / 1,515), 98.9 \%$ (450/455) and 93.8\% (210/224), respectively. The overall quality not sufficient rate due to insufficient fetal fraction (less than 4.0\% fetal fraction or less than 100 copies of cell free fetal DNA) and other not-reportable etiologies rate were $0.9 \%$ and $1.0 \%$, respectively, which was reduced to $0.54 \%$ and $0.1 \%$, respectively after re-sampling. For subchromosomal abnormality detection, Sequenom performed an analytical validation study using genomic DNA mixtures. A clinical validation study, in which the microdeletions/microduplications (MDs) ranged from 3 to 40 Mb, correctly identified 17 of 18 cases with MDs and 156 of 157 unaffected pregnancies [20].

\section{BGI: NIFTY'M}

BGI reported that they tested over 400,000 NIFTY samples collected worldwide [3], and published the largest clinical data involving 146,958 pregnancies in China [5]. The mean maternal age was 30.9 years and the mean gestational age at NIPT was 18.7 weeks. Cytogenetic or phenotypic confirmation of NIPT results was available in 1,066 NIPT positive cases and 111,603 NIPT negative cases. Calculation of NIPT sensitivity, specificity, and positive predictive values was based on this subgroup of the population $(n=112,669)$ with available outcome data. The sensitivity and specificity were $99.17 \%$ and $99.95 \%$ for trisomy 21 , respectively, $98.24 \%$ and $99.95 \%$ for trisomy 18 , respectively, and $100 \%$ and $99.96 \%$ for trisomy 13 , respectively. The false positive rate and positive predictive value were $0.05 \%$ and 92.19\% (720/781) for trisomy 21, 0.05\% and 76.61\% (167/218) for trisomy 18 and $0.14 \%$ and 32.84\% (22/67) for trisomy 13 . There were 9 false negative cases including 6 cases for trisomy 21 and 3 for trisomy 18 . The theoretical positive predictive values were 65.04-94.49\%, 47.44-85.51\% and 57.60-90.05\% for trisomies 21,18 , and 13 , respectively.
When they analyzed the 72,382 high risk and 40,287 low risk pregnancies, there was no significant difference in the overall test performance between these two groups other than a decreased PPV in the low-risk group (94.12\% vs. 81.36\%). A total of 157 false positives and nine false negative results were investigated, and 41 cases were revealed to have biological reasons including 27 cases of maternal copy number variation (CNV), 12 cases of mosaicism, and two cases of confined placental mosaicism. Low fetal fraction $(<3.5 \%)$ was not found in discrepant samples, which could be caused by the relatively late mean gestational age (18.7 weeks) compared to other studies $[5,7,9]$. Among 404 twin pregnancies that had clinical outcome, the sensitivity and specificity for trisomy 21 were $100 \%$ and $99.5 \%$, respectively. Re-sampling was required in 2.18\% of samples due to quality control failure, assay failure, or low fetal fraction; and final test failure rate was $0.098 \%$ [5]. BGI developed a FCAPS (fetal copy number analysis through maternal plasma sequencing) method to detect fetal chromosomal deletions/duplications of $>10 \mathrm{Mb}$ by low coverage WGS (about 0.08-fold). This method was tested in 1,311 maternal plasma with known karyotyping results of the fetus including three cases of $>10 \mathrm{Mb}$ deletion/duplication. All three cases were detected with only one false positive case using the FCAPS method [21].

\section{Illumina Verifi ${ }^{\circledR}$}

Illumina evaluated test performance using a 34,306 clinical cohort [9]. The mean maternal age was 35.4 years and the mean gestational age at NIPT was 14.2 weeks. The sensitivity and specificity were $99.14 \%$ and $99.94 \%$, respectively for trisomy 21 , $98.31 \%$ and $99.90 \%$, respectively for trisomy 18 , and $98.89 \%$ and $99.79 \%$, respectively for trisomy 13 . Positive predictive values were 96.9\% (596/615) for trisomy 21, 86.3\% (208/241) for trisomy 18 , and $79.8 \%$ (71/89) for trisomy 13 in this study group. There were 9 false negative cases including 5 for trisomy 21, 3 for trisomy 18 , and 1 for trisomy 13 . For monosomy $X$, the sensitivity and specificity were 95\% (19/20) and 99\% (483/488), respectively [22]. The technical failure rate was reported as $0.1 \%$ [9].

\section{Conclusion}

The outstanding clinical performance of WGS-based NIPT has been proven by several large clinical studies. We expect the false positive rate could be reduced through genome wide analysis, which can detect false positives due to maternal CNV. Compared to WGS-based NIPT, targeted sequencing-based NIPT 
has the advantage of requiring less sequencing, which reduces costs. Particularly, single nucleotide polymorphism-based NIPT allow additional detection of triploidy, uniparental disomy and consanguinity, and separate evaluation of dizygotic twins. Combining different approaches could achieve optimal NIPT that is accurate and cheap. In the near future, decreasing sequencing costs could increase the worldwide adoption of NIPT and expand the scope of NIPT for detecting other chromosomal imbalances.

\section{References}

1. MaterniT21 ${ }^{\circledR}$ PLUS. [https://laboratories.sequenom.com/providers/ maternit21-plus/\#clinical]

2. VisibiliT. [https://laboratories.sequenom.com/providers/visibilit/]

3. NIFTY. [http://www.niftytest.com/]

4. Verifi ${ }^{\circledR}$. [http://www.illumina.com/clinical/reproductive-genetichealth/clinical-labs/nipt.html]

5. Zhang $H$, Gao $Y$, Jiang F, Fu M, Yuan Y, Guo Y, et al. Non-invasive prenatal testing for trisomies 21, 18 and 13: clinical experience from 146,958 pregnancies. Ultrasound Obstet Gynecol 2015;45:530-8.

6. Dan S, Wang W, Ren J, Li Y, Hu H, Xu Z, et al. Clinical application of massively parallel sequencing-based prenatal noninvasive fetal trisomy test for trisomies 21 and 18 in 11,105 pregnancies with mixed risk factors. Prenat Diagn 2012;32:1225-32.

7. McCullough RM, Almasri EA, Guan X, Geis JA, Hicks SC, Mazloom AR, et al. Non-invasive prenatal chromosomal aneuploidy testing--clinical experience: 100,000 clinical samples. PLoS One 2014;9:e109173.

8. Bianchi DW, Platt LD, Goldberg JD, Abuhamad AZ, Sehnert AJ, Rava RP; MatErnal BLood IS Source to Accurately diagnose fetal aneuploidy (MELISSA) Study Group. Genome-wide fetal aneuploidy detection by maternal plasma DNA sequencing. Obstet Gynecol 2012; 119:890-901.

9. Bhatt S, Parsa S, Snyder H, Taneja P, Halks-Miller M, Seltzer W, et al. Clinical laboratory experience with noninvasive prenatal testing: update on clinically relevant metrics. Poster presented at the 18th International Conference on Prenatal Diagnosis and Therapy; 2014 Jul 20-23; Brisbane. [http://www.vcgs.org.au/downloads/NIPT/ISPD_ Poster_2014_r3_Final_July_2014.pdf]

10. Nygren AO, Dean J, Jensen TJ, Kruse $S$, Kwong W, van den Boom D, et al. Quantification of fetal DNA by use of methylation-based DNA discrimination. Clin Chem 2010;56:1627-35.

11. Ehrich M, Deciu C, Zwiefelhofer T, Tynan JA, Cagasan L, Tim R, et al. Noninvasive detection of fetal trisomy 21 by sequencing of DNA in maternal blood: a study in a clinical setting. Am J Obstet Gynecol 2011;204:205.e1-11.
12. Porreco RP, Garite TJ, Maurel K, Marusiak B; Obstetrix Collaborative Research Network, Ehrich $\mathrm{M}_{1}$ et al. Noninvasive prenatal screening for fetal trisomies 21, 18, 13 and the common sex chromosome aneuploidies from maternal blood using massively parallel genomic sequencing of DNA. Am J Obstet Gynecol 2014;211:365.e1-12.

13. Jensen TJ, Zwiefelhofer T, Tim RC, Džakula Ž, Kim SK, Mazloom $A R$, et al. High-throughput massively parallel sequencing for fetal aneuploidy detection from maternal plasma. PLoS One 2013;8: e57381.

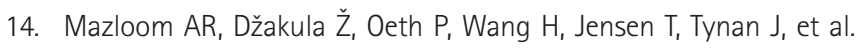
Noninvasive prenatal detection of sex chromosomal aneuploidies by sequencing circulating cell-free DNA from maternal plasma. Prenat Diagn 2013;33:591-7.

15. Kim SK, Hannum G, Geis J, Tynan J, Hogg G, Zhao C, et al. Determination of fetal DNA fraction from the plasma of pregnant women using sequence read counts. Prenat Diagn 2015;35:810-5.

16. Jiang F, Ren J, Chen F, Zhou Y, Xie J, Dan S, et al. Noninvasive Fetal Trisomy (NIFTY) test: an advanced noninvasive prenatal diagnosis methodology for fetal autosomal and sex chromosomal aneuploidies. BMC Med Genomics 2012;5:57.

17. Zheng J, Xu C, Guo J, Wei $Y, G e H_{1}$ Li $X_{1}$ et al. Effective noninvasive zygosity determination by maternal plasma target region sequencing. PLoS One 2013;8:e65050.

18. Futch T, Spinosa J, Bhatt $S$, de Feo E, Rava RP, Sehnert AJ. Initial clinical laboratory experience in noninvasive prenatal testing for fetal aneuploidy from maternal plasma DNA samples. Prenat Diagn 2013; 33:569-74.

19. Sehnert AJ, Rhees B, Comstock D, de Feo E, Heilek G, Burke J, et al. Optimal detection of fetal chromosomal abnormalities by massively parallel DNA sequencing of cell-free fetal DNA from maternal blood. Clin Chem 2011;57:1042-9.

20. Zhao C, Tynan J, Ehrich M, Hannum G, McCullough R, Saldivar JS, et al. Detection of fetal subchromosomal abnormalities by sequencing circulating cell-free DNA from maternal plasma. Clin Chem 2015;61: 608-16.

21. Chen $S$, Lau TK, Zhang $C, X u C_{1}, X u Z$, Hu P, et al. A method for noninvasive detection of fetal large deletions/duplications by low coverage massively parallel sequencing. Prenat Diagn 2013;33:58490.

22. Verinata Health Inc. Analytical validation of the verifi prenatal test: Enhanced test performance for detecting trisomies 21, 18 and 13 and the option for classification of sex chromosome status. Redwood City, CA: Verinata Health, Inc., 2012. [http://www.prenatalsafe.it/pdf/ bibliografia/10_verifi_white_paper_Letter-Size_11-16-2012.pdf] 